\title{
Correction to: Fair Outlier Detection
}

\author{
Deepak P. and Savitha Sam Abraham
}

\section{Correction to:}

Chapter "Fair Outlier Detection" in: Z. Huang et al. (Eds.):

Web Information Systems Engineering - WISE 2020, LNCS 12343, https://doi.org/10.1007/978-3-030-62008-0_31

The original version of this chapter was revised. The first author's name was corrected to Deepak P. 